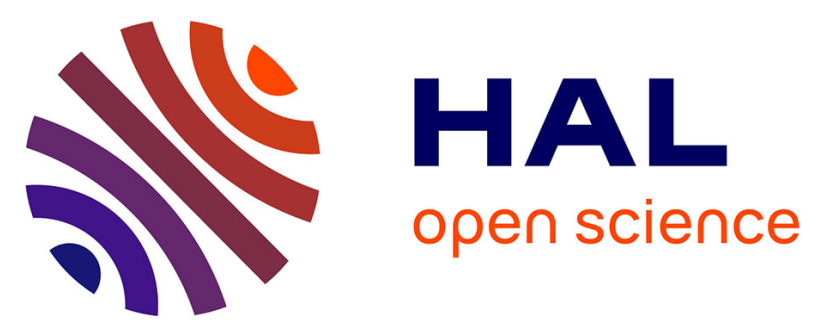

\title{
A comparison of higher-order vertical velocity moments in the convective boundary layer from lidar with in situ measurements and LES
}

\author{
Donald H Lenschow, Marie Lothon, Shane D Mayor, Peter P Sullivan, \\ Guylaine Canut
}

\section{To cite this version:}

Donald H Lenschow, Marie Lothon, Shane D Mayor, Peter P Sullivan, Guylaine Canut. A comparison of higher-order vertical velocity moments in the convective boundary layer from lidar with in situ measurements and LES. Boundary-Layer Meteorology, 2012, 143 (1), pp.107-123. 10.1007/s10546011-9615-3 . hal-01010030

\section{HAL Id: hal-01010030 \\ https://hal.science/hal-01010030}

Submitted on 25 Oct 2021

HAL is a multi-disciplinary open access archive for the deposit and dissemination of scientific research documents, whether they are published or not. The documents may come from teaching and research institutions in France or abroad, or from public or private research centers.
L'archive ouverte pluridisciplinaire HAL, est destinée au dépôt et à la diffusion de documents scientifiques de niveau recherche, publiés ou non, émanant des établissements d'enseignement et de recherche français ou étrangers, des laboratoires publics ou privés. 


\title{
A Comparison of Higher-Order Vertical Velocity Moments in the Convective Boundary Layer from Lidar with In Situ Measurements and Large-Eddy Simulation
}

\author{
Donald H. Lenschow • Marie Lothon • Shane D. Mayor • \\ Peter P. Sullivan • Guylaine Canut
}

\begin{abstract}
We have analyzed measurements of vertical velocity $w$ statistics with the NOAA high resolution Doppler lidar (HRDL) from about $390 \mathrm{~m}$ above the surface to the top of the convective boundary layer (CBL) over a relatively flat and uniform agricultural surface during the Lidars-in-Flat-Terrain (LIFT) experiment in 1996. The temporal resolution of the zenithpointing lidar was about $1 \mathrm{~s}$, and the range-gate resolution about $30 \mathrm{~m}$. Vertical cross-sections of $w$ were used to calculate second- to fourth-moment statistics of $w$ as a function of height throughout most of the CBL. We compare the results with large-eddy simulations (LES) of the CBL and with in situ aircraft measurements. A major cause of the observed case-to-case variability in the vertical profiles of the higher moments is differences in stability. For example, for the most convective cases, the skewness from both LES and observations changes more with height than for cases with more shear, with the observations changing more with stability than the LES. We also found a decrease in skewness, particularly in the upper part of the CBL, with an increase in LES grid resolution.
\end{abstract}

Keywords Higher-order moments · Lidar measurements · Turbulence statistics · Velocity moments · Vertical velocity

D. H. Lenschow ( $\varangle)$ · P. P. Sullivan

National Center for Atmospheric Research, P. O. Box 3000, Boulder, CO 80307-3000, USA

e-mail: lenschow@ucar.edu

M. Lothon · G. Canut

Centre de Recherches Atmosphériques, 8 route de Lannemezan, 65300 Campistrous, France

S. D. Mayor

Department of Physics, California State University, Chico, 400 West First Street, Chico, CA 95929, USA

Present Address:

G. Canut

Institute for Climate and Atmospheric Science, School of Earth and Environment, University of Leeds, Leeds LS2 9JT, UK 


\section{Introduction}

Higher-order velocity statistics can provide useful information about the structure of turbulence in the convective boundary layer (CBL). Profiles of velocity variance are commonplace, but say nothing about how the turbulence departs from, e.g. a Gaussian distribution. Moments higher than two can provide this information, but with a considerable increase in random (sampling) error (Lenschow et al. 1994). That is, for the same relative statistical significance, a considerably longer measurement time is required for moments greater than two. This is one reason why there are few measurements of moments greater than two reported in the literature. Doppler lidar is one tool that can be used to address the sampling issue. The lidar can point in a particular direction for long periods of time and simultaneously measure the radial velocity component of aerosol particles in each sampling volume along the beam.

In the CBL, the probability distribution of the vertical air velocity $w$ is of particular interest since the fluctuations at long wavelengths are attenuated, and thus they do not result in unpredictable spread of the probability distribution, as is the case for horizontal velocity components and scalars. Furthermore, the distribution is significantly non-Gaussian, so that the information is useful for understanding the structure of $w$ fluctuations in the CBL.

We discuss in this article measurements of $w$ with the $2.022 \mu \mathrm{m}$ wavelength high-resolution Doppler lidar (HRDL), developed by the NOAA Environmental Technology Laboratory and described by Grund et al. (1998), during the Lidars-in-Flat-Terrain (LIFT) experiment. Details of the experiment and of the corollary experiment, the Flatlands experiment, are presented in Angevine et al. (1998) and Cohn et al. (1998). Many auxiliary measurements were also obtained during the LIFT and Flatland experiments. Profiles of mean horizontal wind $U$ were obtained from a wind profiler located at Sadorus, Illinois, USA, about $5 \mathrm{~km}$ from the HRDL. The mean potential temperature and water vapour mixing ratio were estimated from radiosonde soundings nearest in time to the selected periods, about $5 \mathrm{~km}$ from the HRDL. Studies of vertical coherence (Lothon et al. 2006) and spectra (Lothon et al. 2009) using this dataset have previously been reported. Recently, Ansmann et al. (2010) also reported lidar measurements of $w$ statistics, which generally showed turbulence statistics similar to those observed here, although they did not normalize their results.

The HRDL was pointed straight up for extended periods of time on 12 days for a total of $110 \mathrm{~h}$ to measure $w$ in the CBL between 26 July and 22 August 1996. It operated at a pulse repetition rate of $200 \mathrm{~s}^{-1}$ in the zenith-pointing mode over level farmland (a patchwork of corn and soybean fields) in central Illinois, U.S.A. The spatial resolution was about $30 \mathrm{~m}$ and the distance from the lidar to the first detectable velocity signal ('dead zone') was about $390 \mathrm{~m}$. The lidar vertical range generally did not extend beyond the top of the CBL because of the reduced aerosol content of the free troposphere. However, above about $z=0.9 z_{i}$, where $z_{i}$ is the CBL depth, the lidar signal occasionally dropped out, likely due to the entrainment of low-backscatter parcels from the overlying free troposphere, which limits the effective vertical range of the $w$ measurement.

The resulting vertical cross-sections were used to calculate moments of $w$ as a function of height $z$ with unprecedented vertical resolution throughout the mid-region of the CBL. We compare the observed statistics for 11 cases (one case was rejected due to excessive noise in the data) centred about mid-afternoon (from about 1300 to $1600 \mathrm{~h}$ local time or CST) with previous formulations based on both measurements and numerical simulations, and discuss the differences, both on an averaged and a case-by-case basis.

The observations used for comparison were obtained from both aircraft and tethered balloons. Aircraft measurements from the Air Mass Transformation Experiment (AMTEX), which occurred over the East China Sea during cold air outbreaks in February 1975, are 
reported by Lenschow et al. (1980). For this case, a baroclinic CBL is well capped because of the subsiding relatively warm air over the cold continental air streaming out of central Asia that overlies the warm north-eastward-flowing Kuroshio ocean current. We also used aircraft measurements from the African Monsoon Multidisciplinary Analysis (AMMA) (Redelsperger et al. 2006). During AMMA in 2006, the French ATR aircraft intensively probed the Sahelian CBL. We consider here eight flights conducted during the pre-monsoon period (June and early July 2006) above flat areas in the vicinity of Niamey, Niger (Canut et al. 2010). During this period, the CBL was forced primarily by intense surface heating and secondarily by significant wind shear across the CBL top-between the south-westerly monsoon flow in the CBL and the overlying easterly Saharan flow. The tethered balloon measurements were obtained over relatively flat cropland in north-western Minnesota, USA, and reported by Kaimal et al. (1976).

In comparing the averaging process for LIFT measurements to that for aircraft measurements, we note that the use of Taylor's hypothesis to convert the time series into the spatial domain, in which, for a horizontal mean wind speed, $U=5 \mathrm{~m} \mathrm{~s}^{-1}$, a 2-h period is equivalent to a 36-km flight path. The AMTEX aircraft averaging time was $30 \mathrm{~min}$, or $180 \mathrm{~km}$ for an airspeed of $100 \mathrm{~m} \mathrm{~s}^{-1}$. The AMMA sample lengths were 30-40 km long (Canut et al. 2010). From the results of Lothon et al. (2006), the integral scale of $w$ in the vertical is about $0.3 z_{i}$. Therefore, we obtain roughly three simultaneous independent estimates of $w$ from the lidar, so that from a time-averaging perspective, in AMMA the airplane and the lidar sampling lengths are similar. On the other hand, the lidar measures all levels simultaneously, and so enables us obtain more detail in the profiles of turbulence statistics.

We also compare the observational results with very high-resolution large-eddy simulations (LES) of the convective daytime boundary layer described in Sullivan and Patton (2011). From their database of solutions with spatial resolution varying from $32^{3}$ to $1024^{3}$, we focus on the simulations performed with $512^{3}$ gridpoints. In this simulation, the LES computational domain is $\left(L_{x}, L_{y}, L_{z}\right)=(5120,5120,2040) \mathrm{m}$ and the mesh spacing is $\left(\triangle_{x}, \triangle_{y}, \triangle_{z}\right)=(10,10,4) \mathrm{m}$. External inputs to the LES are roughness length $z_{\mathrm{o}}=0.1 \mathrm{~m}$, Coriolis parameter $f=1 \times 10^{-4} \mathrm{~s}^{-1}$ and initial inversion height of $1024 \mathrm{~m}$. Three different combinations of surface virtual temperature flux $\langle w \theta\rangle_{0}$ and geostrophic wind $U_{g}$ are considered: the 'most convective' case with $\left(\langle w \theta\rangle_{0}, U_{g}\right)=\left(0.24 \mathrm{~K} \mathrm{~m} \mathrm{~s}^{-1}, 1 \mathrm{~m} \mathrm{~s}^{-1}\right)$; a 'moderately convective' case with $\left(\langle w \theta\rangle_{0}, U_{g}\right)=\left(0.24 \mathrm{~K} \mathrm{~m} \mathrm{~s}^{-1}, 10 \mathrm{~m} \mathrm{~s}^{-1}\right)$; and the 'least convective' case with $\left(\langle w \theta\rangle_{0}, U_{g}\right)=\left(0.06 \mathrm{Km} \mathrm{s}^{-1}, 10 \mathrm{~m} \mathrm{~s}^{-1}\right)$. A measure of the balance between thermal and shear forcings is the bulk stability parameter $\zeta=-z_{i} / L_{\mathrm{o}}$ with $L_{\mathrm{o}}$ the Obukhov length. For the three LES cases, (most, moderate, least) convective, $\zeta=(684,19.5,5.9)$, respectively. The simulations are initiated from cold starts with random perturbations in the potential temperature field in the lowest $100 \mathrm{~m}$ of the domain. The computations are advanced in time for more than $25 \tau$, where the large-eddy turnover time $\tau=z_{i} / w_{*}$ and $w_{*}$ is the Deardorff convective velocity scale,

$$
w_{*}=\left(\frac{g}{T}\langle w \theta\rangle_{0} z_{i}\right)^{1 / 3},
$$

where $g$ is the acceleration due to gravity and $T$ is the mean CBL temperature. As is common practice, turbulence statistics are obtained from averages over horizontal planes at each $z$ and in time. The temporal averaging spanned the last 15 turnover times of the simulations. Sullivan and Patton (2011) show that velocity spectra from the $512^{3}$ simulation display an extended inertial subrange and the low-order statistical moments (turbulence variances and fluxes, and entrainment rate) have numerically converged as compared with simulations with 
coarser and finer resolutions. The numerical solution algorithm and subgrid-scale model are described in Moeng (1984) and Sullivan et al. (1994, 1996).

\section{Comparisons of Observations with Large-Eddy Simulation}

In this section, we show profiles of the second- through fourth-order moments of $w$. The moments from LIFT are calculated over the entire time series, so that the averaging distance varies, depending on the mean wind speed $U$ and the length of the time series, from $24 z_{i}$ to $96 z_{i}$. The moments have all been corrected for uncorrelated noise using the technique developed by Lenschow et al. (2000), but the noise contribution is small. Table 1 lists the defining parameters for the 11 LIFT cases, including details of both the mean and turbulence structure; a more detailed table that also describes the transition to the overlying free troposphere for these cases is presented in Lothon et al. (2009).

\subsection{Variance and Dissipation}

The left panel of Fig. 1 shows the vertical velocity variance $\left\langle w^{2}\right\rangle$ as a function of normalized height $z_{*}=z / z_{i}$ for all eleven LIFT cases. In this and the succeeding figures, the red symbols are the most convective cases $(\zeta \geq 30$ - a total of five cases) and the blue the least unstable $(\zeta<30-$ a total of six cases). The lines without symbols are weighted averages; that is, they were obtained by applying a weighting factor to each case to take into account the sample length of each case, which is the product of $U$ times the sample period. The lines without symbols all show a maximum variance at about $0.3 z_{i}-0.4 z_{i}$, which is consistent with previous observations (e.g. Lenschow et al. 1980). (The averaged LIFT values at the bottom and top of the profile are influenced by the 'dropout' of some cases due to the variation in range arising from the height normalization process; thus, the changes in slope at the ends of the lines in this and subsequent figures are anomalous.) Not surprisingly, on average, the most unstable cases have the largest variances.

The right panel of Fig. 1 shows the variances normalized by $w_{*}^{2} ;\langle w \theta\rangle_{0}$ was obtained from Flux-PAM (Portable Automated Mesonet) surface-flux-measurement stations deployed in the Flatlands experiment (Militzer et al. 1995) within a few kilometres of the HRDL site.

Table 1 Mean characteristics of the 11 LIFT cases considered here

\begin{tabular}{|c|c|c|c|c|c|c|c|c|}
\hline $\begin{array}{l}\text { Date } \\
(1996)\end{array}$ & Symbol & $\begin{array}{l}\text { Time period } \\
\text { (UTC) }\end{array}$ & $z_{i}(\mathrm{~m})$ & $\begin{array}{l}U \\
\left(\mathrm{~ms}^{-1}\right)\end{array}$ & $\begin{array}{l}w_{*} \\
\left(\mathrm{~ms}^{-1}\right)\end{array}$ & $\begin{array}{l}u_{*} \\
\left(\mathrm{~ms} \mathrm{~s}^{-1}\right)\end{array}$ & $\zeta$ & $\chi$ \\
\hline 2 Aug & + & $1700-2000$ & 1350 & 3.0 & 1.50 & 0.16 & 346 & 0.47 \\
\hline 4 Aug & $*$ & $1700-2000$ & 1230 & 5.2 & 1.17 & 0.35 & 15 & 0.26 \\
\hline 5 Aug & $\square$ & $1700-2000$ & 1140 & 8.6 & 1.32 & 0.52 & 6 & 0.28 \\
\hline 6 Aug & $\triangle$ & $1800-2100$ & 1170 & 7.8 & 1.28 & 0.46 & 8 & 0.20 \\
\hline 7 Aug & $\diamond$ & $1800-2100$ & 1110 & 5.6 & 1.23 & 0.39 & 13 & 0.28 \\
\hline 10 Aug & $\nabla$ & $1700-2100$ & 1650 & 2.2 & 1.51 & 0.39 & 205 & 0.21 \\
\hline $12 \mathrm{Aug}$ & $\circ$ & $1800-2100$ & 1410 & 4.8 & 1.46 & 0.19 & 30 & 0.41 \\
\hline $16 \mathrm{Aug}$ & $\bullet$ & $1800-2100$ & 1350 & 2.2 & 1.62 & 0.34 & 233 & 0.17 \\
\hline 19 Aug & $\square$ & $1800-2000$ & 1200 & 7.2 & 1.19 & 0.19 & 8 & 0.21 \\
\hline 20 Aug & $\diamond$ & $1800-2100$ & 930 & 6.8 & 1.13 & 0.43 & 8 & 0.05 \\
\hline $21 \mathrm{Aug}$ & $\Delta$ & $1800-2100$ & 1020 & 3.4 & 1.11 & 0.26 & 32 & 0.13 \\
\hline
\end{tabular}

Local time $=\mathrm{UTC}-6 \mathrm{~h}, w_{*}$ is the convective velocity scale, $u_{*}$ is the friction velocity, $\zeta=-z_{i} / L_{\mathrm{O}}$, where $L_{\mathrm{O}}$ is the Obukhov length, and $\chi$ is the cloud fraction 
(a)

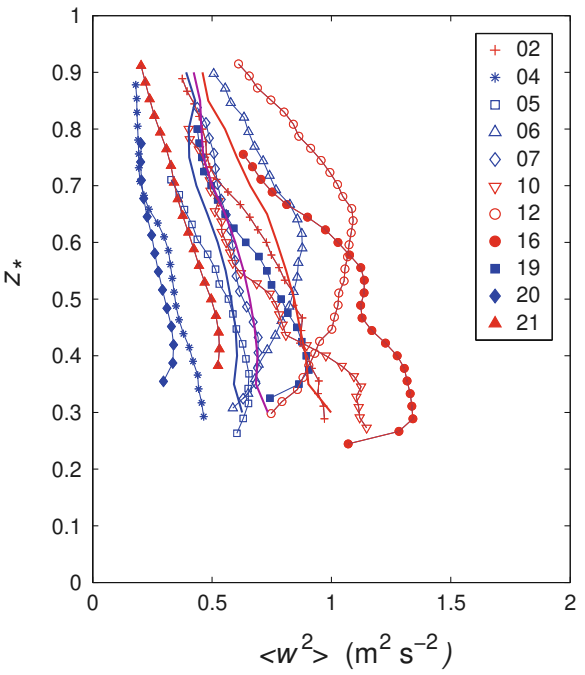

(b)

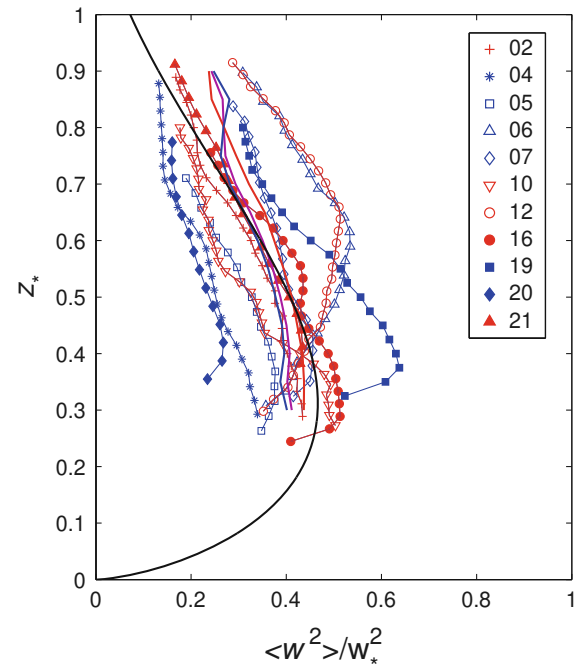

Fig. 1 Profiles of vertical velocity variance measured by HRDL in the CBL during LIFT. The symbols identify the dates (in August, 1996) of the 11 cases, as described in Table 1. The smoothed coloured lines are averages of the observations; the red line and symbols are the most unstable cases, the blue line and symbols the least unstable, and the magenta line the overall weighted average. The solid black line in the right panel is the empirical curve, $\left\langle w^{2}\right\rangle / w_{*}^{2}=1.8 z_{*}^{2 / 3}\left(1-0.8 z_{*}\right)^{2}$ obtained by Lenschow et al. (1980)

As expected, the normalization collapses the cases so that, although there is considerable scatter, on average, there is no significant variation with $\zeta$. Using the analysis of Lenschow et al. (1994), the average relative standard error due to finite sampling over all the cases in the middle of the CBL, corrected for the added contributions of skewness and kurtosis, is about $15 \%$. The mid-CBL average relative standard error for the measured $\left\langle w^{2}\right\rangle$ is about $38 \%$; for $\left\langle w^{2}\right\rangle / w_{*}^{2}$ it is about $25 \%$. Thus, about $10 \%$ of the scatter in the measured $\left\langle w^{2}\right\rangle / w_{*}^{2}$ is due to real case-to-case variability that is not removed by the $w_{*}$ scaling (and about $13 \%$ is removed by the normalization). The plot of $\left\langle w^{2}\right\rangle / w_{*}^{2}$ in Fig. 1 shows that some residual variation still exists in the normalized variance due to stability, which is one source of this 'extra' scatter.

Three cases have variances that are elevated compared to the others: Days 6 and 12 have elevated variance in the upper CBL, and Day 19 is elevated just below the middle of the CBL. Lothon et al. (2009) found that Day 6 had an anomalously large normalized alongwind integral scale, $l_{w} / z_{i}$, which was as much as $3-5$ times any other case. They found that the spectra for this case had a secondary spectral peak at a wavelength of about $10 \mathrm{~km}$ that becomes predominant in the middle and upper part of the CBL, and suggest that this was the result of mesoscale motions-possibly longitudinal roll circulations-since the Weckwerth (1999) criterion for roll circulation $(\zeta<25)$ was met. Similarly, Day 19 also had $\zeta<25$ and a secondary spectral peak at about $7 \mathrm{~km}$. Day 12 was different; on this more convective day $(\zeta \approx 30)$, Lothon et al. (2009) found a very regular modulation in cloud cover with a spacing of about $4 \mathrm{~km}$ and updraft velocity into the cumulus cloud bases, which again generated 'extra' variance at somewhat longer wavelengths-in this case also about $4 \mathrm{~km}$ - than for purely surface-flux-driven cases. None of the other cases showed such a direct correlation between clouds and updraft velocity. 
These results show that in the real world, mixed-layer CBL scaling does not explain all the observed variation in variance among all the cases. Although this region is a patchwork of corn and soybean fields, Angevine et al. (1998) found that sensible heat fluxes were about the same over both crops during the observational period. Earlier Hicks and Wesely (1981) found, also over central Illinois in mid-summer at midday, little difference between sensible heat fluxes over corn and soybeans, infrared surface temperature differences within $0.02^{\circ} \mathrm{C}$, and friction velocity over corn only about $17 \%$ greater than over soybeans. Therefore, we conclude that surface variability is likely not a major factor in explaining the elevated variance. Other sources could be case-to-case differences in wind shear (both within the CBL and across the CBL top), inversion strength, free tropospheric lapse rate, and entrainment rate (estimated by Lothon et al. (2009) using the Tennekes (1973) model and evaluating the turbulence kinetic energy budget with estimates of dissipation from the $w$ spectra). Using the results of Lothon et al. (2009), however, we could see no obvious correlations between these variables and enhanced variance. Later, we show that the higher-moment statistics for elevated variance cases are not anomalous; that is, the mesoscale processes that produce extra variance do not have a noticeable impact on the higher-moment statistics.

We show comparisons of the variance from the averaged LIFT profiles with the LES results of Sullivan and Patton (2011) and the AMMA results (Canut et al. 2010) in Fig. 2. The LES estimates of the variance are computed using the resolved $w$. The subgrid-scale contribution to $\left\langle w^{2}\right\rangle$, approximately $2 / 3$ the subgrid-scale energy, is small everywhere except very near the surface. The values of $\zeta$ for the three LES cases presented in the figures are as

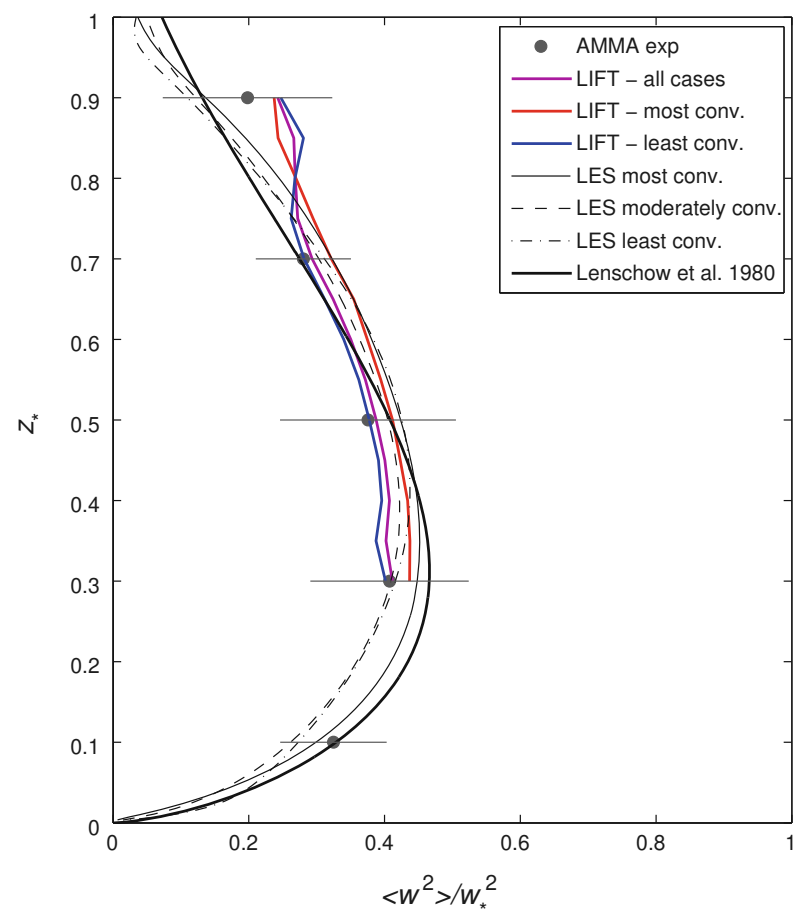

Fig. 2 Profiles of normalized vertical velocity variance in the CBL showing the LIFT and AMMA observations, the LES results, and the empirical curve of Lenschow et al. (1980) 


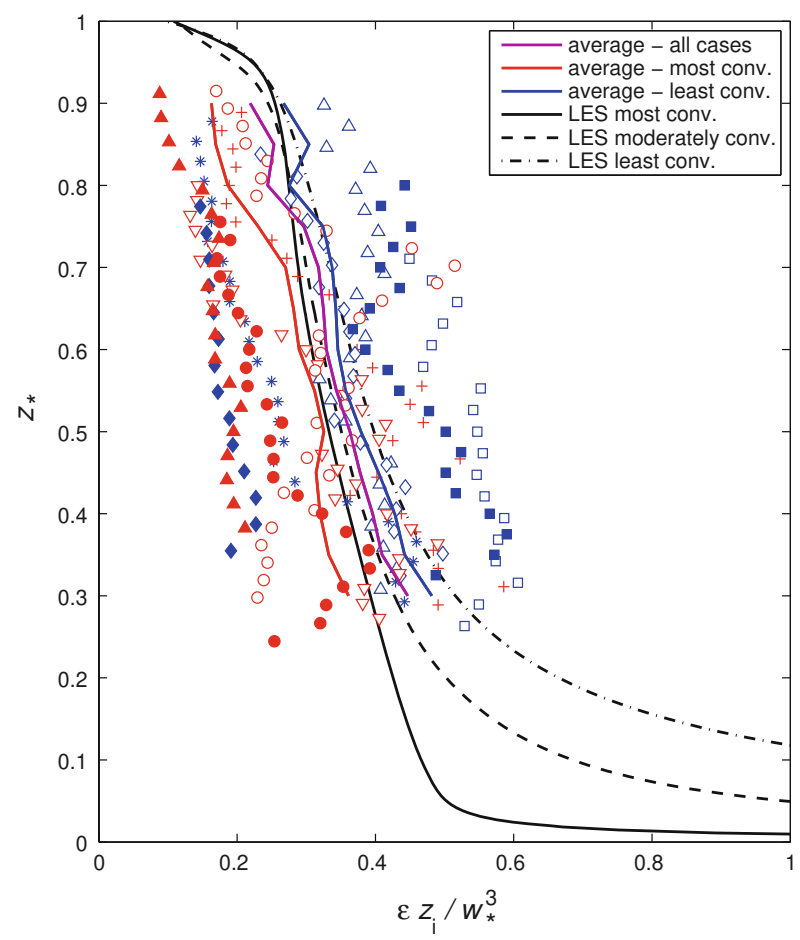

Fig. 3 Profiles of normalized dissipation in the CBL obtained from the inertial subrange of the transverse velocity spectrum over a range of $30<k z_{i}<80$, as described in Lothon et al. (2009). The smoothed coloured lines are averages of the observations and the black curves are LES results (Sullivan and Patton 2011)

follows: the least convective (largest shear), $\zeta=5.9$, the moderately convective, $\zeta=19.5$ and the most convective (least shear), $\zeta=684$. This range encompasses all the observed values encountered in LIFT. The LES results also show little variation with $\zeta$, and agree well with the general shape of the LIFT profiles and with the aircraft observations of Lenschow et al. (1980), which follow the relation plotted in Fig. 2,

$$
\frac{\left\langle w^{2}\right\rangle}{w_{*}^{2}}=1.8 z_{*}^{2 / 3}\left(1-0.8 z_{*}\right)^{2} .
$$

The aircraft observations also agree with the tethered-balloon observations reported by Kaimal et al. (1976).

Another useful comparison can be made between the normalized turbulence energy dissipation $\epsilon z_{i} / w_{*}^{3}$ from LIFT with the LES results, shown in Fig. 3. The LIFT estimates of $\epsilon z_{i} / w_{*}^{3}$ are obtained from the inertial subrange of the transverse velocity spectrum over a range of $30<k z_{i}<80$, where $k$ is wavenumber, as described in Lothon et al. (2009). Basically, $\epsilon z_{i} / w_{*}^{3}$ is proportional to the $3 / 2$ power of the band-pass-filtered $w$ variance. Dissipation in the LES is computed from the inertial subrange parametrization $\epsilon=C_{\epsilon} e^{3 / 2} / \triangle_{f}$ where $e$ is the subgrid-scale energy, $\triangle_{f}$ is the LES filter width and $C_{e} \approx 0.93$ is a modelling constant (Moeng and Wyngaard 1988).

On average, we see good agreement with the LES results, including a similar increase in $\epsilon z_{i} / w_{*}^{3}$ with a decrease in $z_{i} / L$; that is, in cases with more shear, $\epsilon z_{i} / w_{*}^{3}$ is larger, as expected, 
since the normalization does not include the contribution to turbulence energy from shear. This provides some assurance of the viability of using the lidar measurements to estimate $\epsilon$, as well as a test of how well the LES and the observations resemble each other. Further assurance is provided by O'Connor et al. (2010) who have examined in detail errors in lidar measurements of $\epsilon$ with a similar Doppler lidar and compared the lidar measurements with balloon-borne in situ $\epsilon$ measurements in the CBL; they also found good agreement. There is a suggestion of a slightly smaller dissipation for $z_{*}>0.7$ for the LIFT observations in the most convective case. One possible reason for this would be a larger negative entrainment flux of buoyancy in the observed cases compared to the LES, as suggested by Lothon et al. (2009).

\subsection{Skewness and Kurtosis}

The left panel of Fig. 4 shows the normalized third moments of $w$ for LIFT. We see considerably more scatter than for $\left\langle w^{2}\right\rangle / w_{*}^{2}$, and a definite increase in the third moment with increasing instability, which seems not to have been previously documented. Two days stand out as having larger normalized third moments than the others: Day 12, which, as noted earlier, was noticeably affected by cloud updrafts and Day 16, which was the most convective case. We note, however, that Day 16, is a light-wind case, which implies that the sampling statistics are poorer than for the other cases. Large scatter is also evident in the right panel of Fig. 4, which shows profiles of the $w$ skewness, defined by

$$
S \equiv \frac{\left\langle w^{3}\right\rangle}{\left\langle w^{2}\right\rangle^{3 / 2}} .
$$

(a)

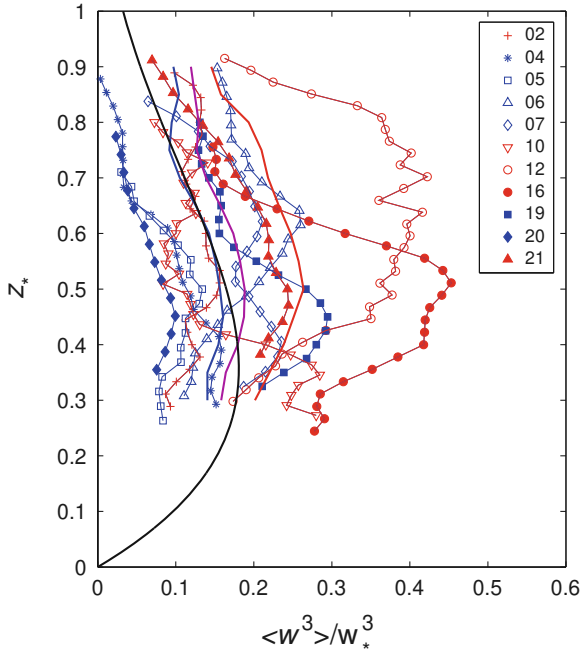

(b)

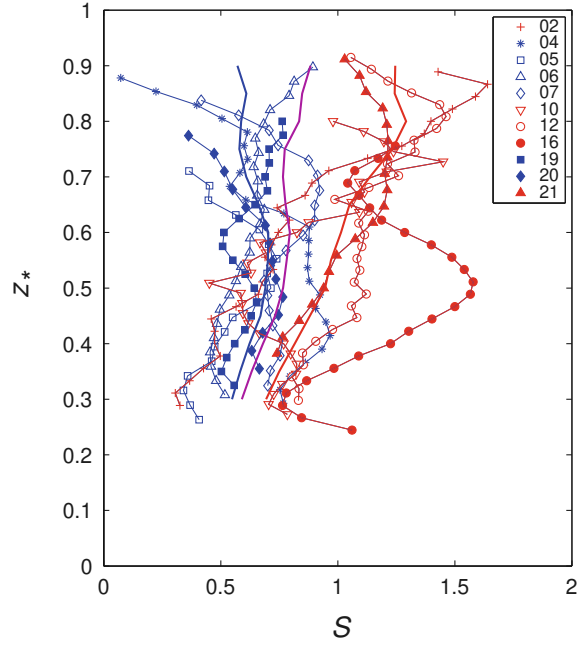

Fig. 4 The left panel shows profiles of the third moment of $w$ normalized by $w_{*}$ and the right panel shows profiles of $w$ skewness. The symbols are lidar measurements from LIFT defined in Table 1 . The lines without symbols are the averages of the observations, with blue the least unstable, red the most convective, and magenta the overall average. The solid black curve is an empirically based formulation $\left\langle w^{3}\right\rangle / w_{*}^{3}=1.2 z_{*}\left(1-0.7 z_{*}\right)^{3}$ 


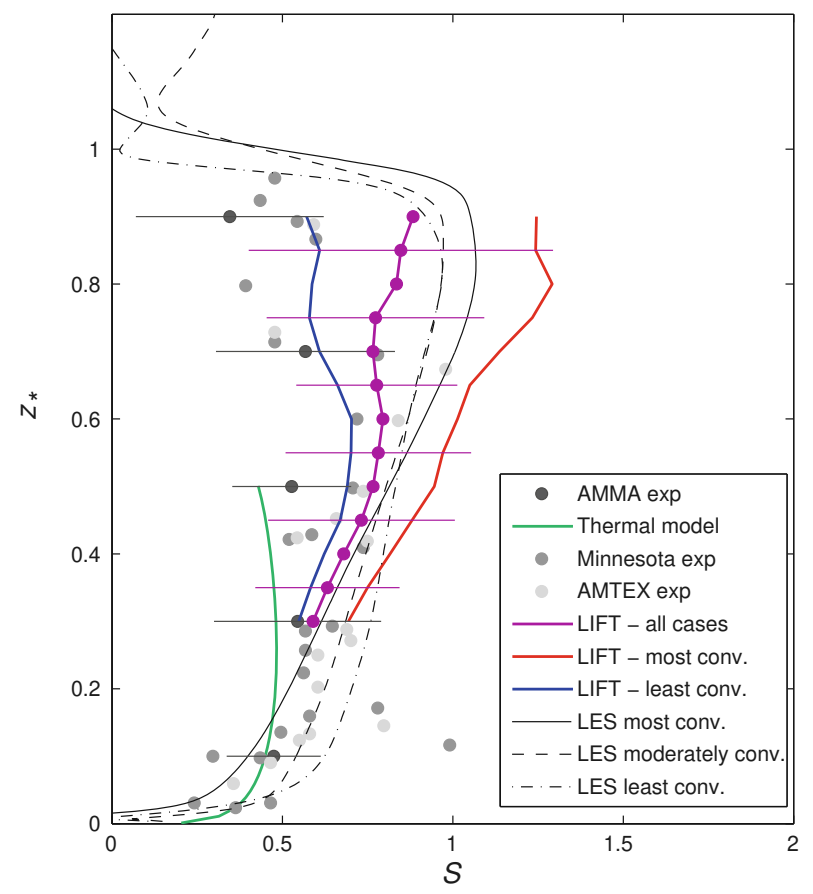

Fig. 5 Profiles of vertical velocity skewness in the CBL. The symbols are observations from previous experiments, as described by Moeng and Rotunno (1990). The black circles intersected with horizontal lines (which indicate the standard deviation of the measurements) are from AMMA (Redelsperger et al. 2006), the dark grey circles are from the Minnesota experiment (Wyngaard 1988), the light grey circles are from AMTEX (Lenschow et al. 1980) and the coloured lines are the averaged LIFT observations (the horizontal magenta lines through the magenta circles are the standard deviations of the LIFT observations). The thin lines are LES results (Sullivan and Patton 2011) and the green line is the thermal model of $S$

Skewness is a measure of the asymmetry of the $w$ probability distribution. Again there is considerable scatter and a definite increase of $S$ with increasing instability. The only case that stands out here is Day 16, which has a maximum of $S \approx 1.5$ in the middle of the CBL; but again, this case has the poorest sampling statistics. On average, these results, including the scatter, are similar to aircraft observations reported by Lenschow et al. (1980, 1994), and Gryanik and Hartmann (2002). Figure 4 also shows an empirical formulation $\left\langle w^{3}\right\rangle / w_{*}^{3}=1.2 z_{*}\left(1-0.7 z_{*}\right)^{3}$ based on Gryanik and Hartmann (2002), but with the coefficient increased from unity to 1.2 to better approximate the results of Lenschow et al. (1980). We see good agreement with the average of the LIFT results; generally, the results of Gryanik and Hartmann (2002) are larger than the LIFT results, even though their range of values of $z_{i} / L, 10-20$, is in our 'least unstable' category.

Figure 5 shows both the observations and the LES results. We see that, on average, the modelled and LIFT values of $S$ are in good agreement up to about $z_{*} \approx 0.5$. However, above that level, the LIFT observations vary considerably with stability. In contrast, the LES results show little variation with stability. The LES results lie between the most convective cases, which continue to increase monotonically with height, and the overall average. The least convective LIFT cases decrease somewhat with height and are in close agreement with previous observations and AMMA observations where we see a maximum of $S \approx 0.6-0.7$ 


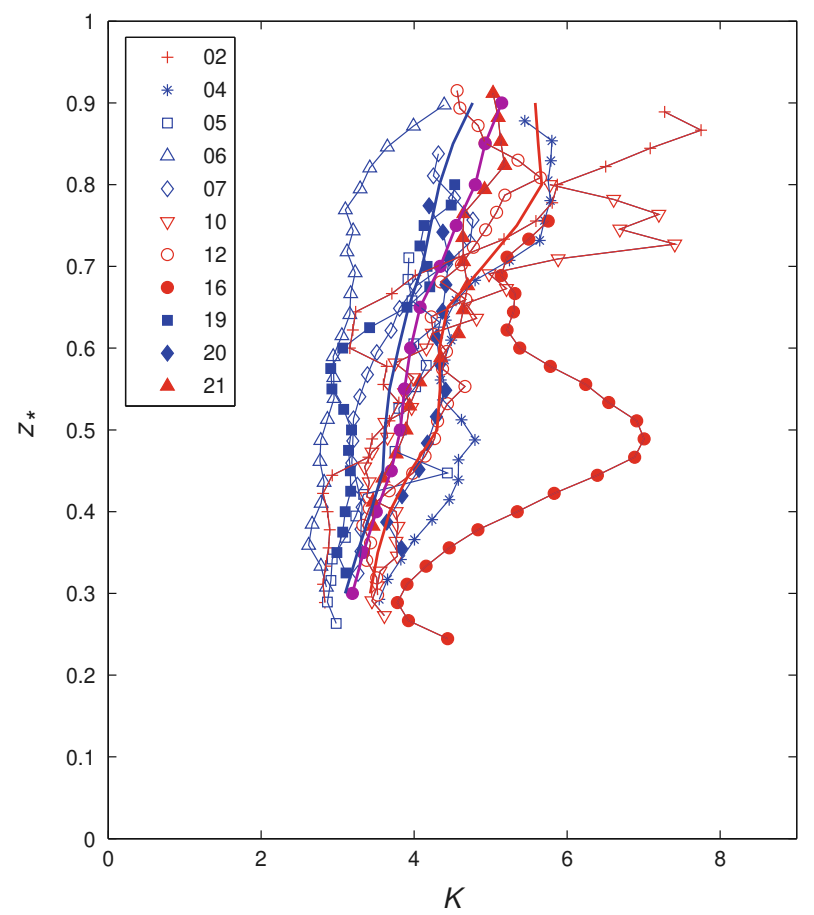

Fig. 6 Profiles of vertical velocity kurtosis in the CBL. The symbols are lidar measurements from LIFT defined in Table 1. The lines without symbols are averages of the observations, with blue the least unstable, red the most convective; the magenta line with circles is the overall average

in the middle of the CBL and a slight decrease with height above. The large values of $S$ for the most convective cases seem not to have been documented previously, perhaps because previous results did not distinguish the most convective cases from an overall average profile of $S$ in the CBL.

Figure 6 shows profiles of the $w$ kurtosis, defined by

$$
K \equiv \frac{\left\langle w^{4}\right\rangle}{\left\langle w^{2}\right\rangle^{2}},
$$

which is a measure of the 'peakedness' of the $w$ probability distribution. Large $K$ is a characteristic of intermittent turbulence. Again, the LIFT results show considerable scatter. (Again, the most convective (lightest wind) case with the poorest sampling statistics, Day 16, has a considerably larger $K$ in the middle of the CBL, similar to $S$ for this case.) Figure 7 shows that both the LES and the averaged LIFT results gradually increase with height, from a value close to the Gaussian $K=3$ up to $z_{*} \approx 0.4$, to values $>4$ in the upper part of the CBL. The LIFT observations again show more variation with stability than the LES results, with the most convective $K$ approaching 6 . We were not able to discern any definitive relationships between $S$ and $K$ on the one hand, and cloud cover, wind shear, inversion strength, free tropospheric lapse rate and entrainment rate on the other hand. This does not preclude the existence of such relationships, however, due to the limited number of cases and the approximations and measurement errors involved in estimating the forcing variables. 


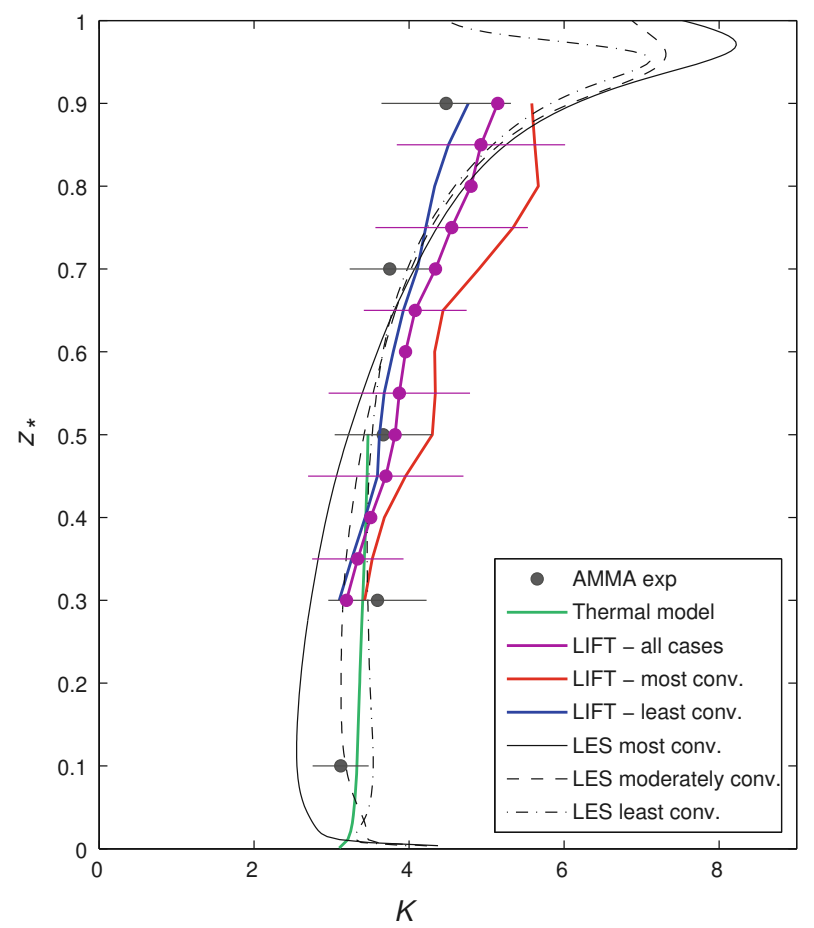

Fig. 7 Profiles of vertical velocity kurtosis in the CBL. The symbols are lidar measurements from LIFT defined in Table 1. The coloured lines are averages of the observations, with blue the least unstable, red the most convective, and magenta the overall average. The horizontal magenta lines through the magenta circles are the standard deviation of the LIFT observations. The thin lines are LES results (Sullivan and Patton 2011) and the green line is the thermal model of $S$

\section{Effects of Averaging}

In this section, we examine the effects of the spatial averaging of observations and reduced resolution of LES on the moments. Figure 8 shows the effects on variance. For this case, the LIFT observations have been averaged over two $15-\mathrm{m}$ gates and four 1-s time intervals (except for Case $2(2 \mathrm{~s})$, and Cases 5 and $10(5 \mathrm{~s})$ ), and the LES resolution has been reduced from $512^{3}$ to $128^{3}$ grid points. We see that the reduced LES resolution has little effect on the LES variance, but the spatial averaging has a significant effect on the LIFT variance at the lower levels- - for the most convective cases, the variance is reduced by about $10 \%$, increasing to about $16 \%$ for the least convective cases. We are not sure of the reason behind this. Perhaps this is the result of the decrease in eddy size approaching the surface (Lothon et al. 2006). In contrast, Fig. 9 shows that there is almost no effect on the spatially-averaged LIFT $S$, while the reduced LES resolution makes a considerable difference in $S$-resulting in a larger $S$ in the upper part of the CBL and a smaller $S$ near the surface.

Sullivan and Patton (2011) formulate an expression for subgrid-scale skewness in LES that depends on both the subgrid-scale second- and third-order moments. They find that the subgrid-scale contribution to $S$ is larger than unity in the surface layer and less than unity at the CBL top. Since LES typically closes the equations at the second-moment level, coarse-mesh LES underpredicts $S$ in the surface layer and overpredicts $S$ at the inversion. Subgrid-scale 


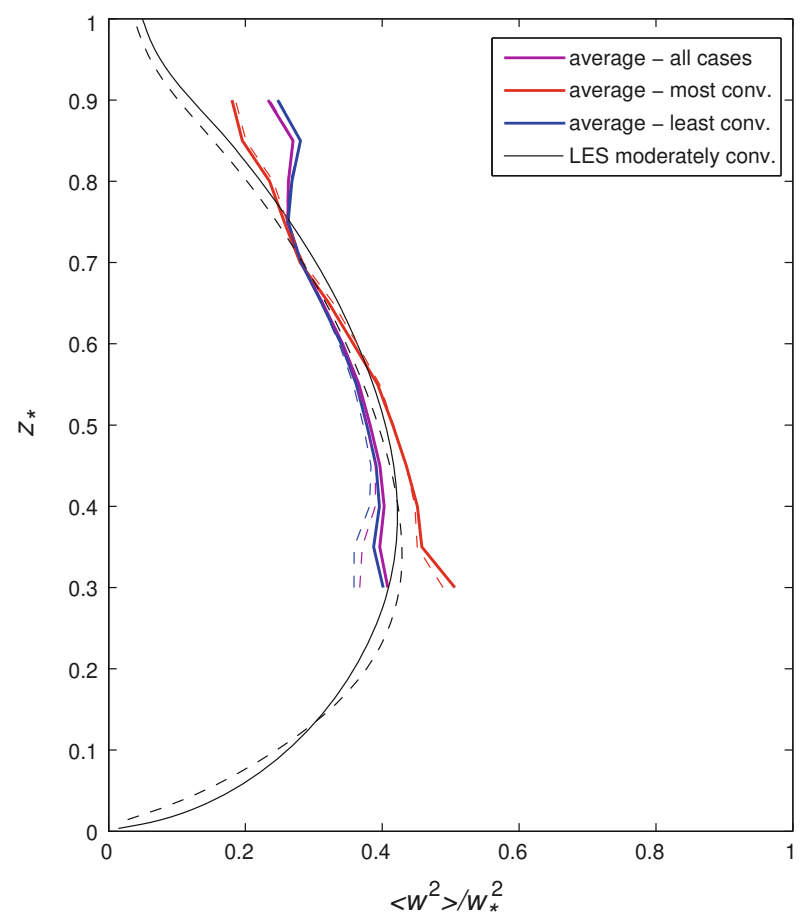

Fig. 8 Effect of spatial averaging on the $w$ variance from LIFT observations and the effect of reduced resolution on LES—-both are indicated by the dashed lines

contributions to fourth-order moments also affect the LES estimates of $K$, especially near the surface and in the upper part of the boundary layer. Indeed, Fig. 10 shows that a similar pattern is true for $K$ : a small effect of averaging on observed $K$, an increase in $K$ in the upper CBL and a decrease in the lower CBL for reduced LES resolution. The larger effect of averaging on $\langle w\rangle^{2}$ than $S$ and $K$ is likely a result of the normalization of the third and fourth moments by the variance; that is, any reduction in the third and fourth moments due to averaging is compensated by a similar loss in variance.

Sullivan and Patton (2011) found that a grid resolution of $256^{3}$ is required for second-order moments to be free of subgrid-scale effects. For this LES configuration, this is equivalent to the ratio $z_{i} /\left(C_{\mathrm{s}} \Delta f\right) \approx 300$, where the Smagorinsky constant $C_{\mathrm{s}}=0.18$ and $\Delta f$ is the filter width. For higher-order moments, the ratio needs to be even larger.

\section{Skewness Versus Kurtosis}

\subsection{Comparisons with Previous Parametric Models}

Figure 11 shows a parametric curve of skewness vs. kurtosis for the LIFT data, as well as the AMMA aircraft data and the LES results. In addition, we show three parametrized curves for comparison. Lenschow et al. (1994) modelled a non-Gaussian process by modifying a Gaussian process $\tilde{z}(t)$ with variance $\left\langle\tilde{z}^{2}\right\rangle$ and an exponential autocorrelation function such that 


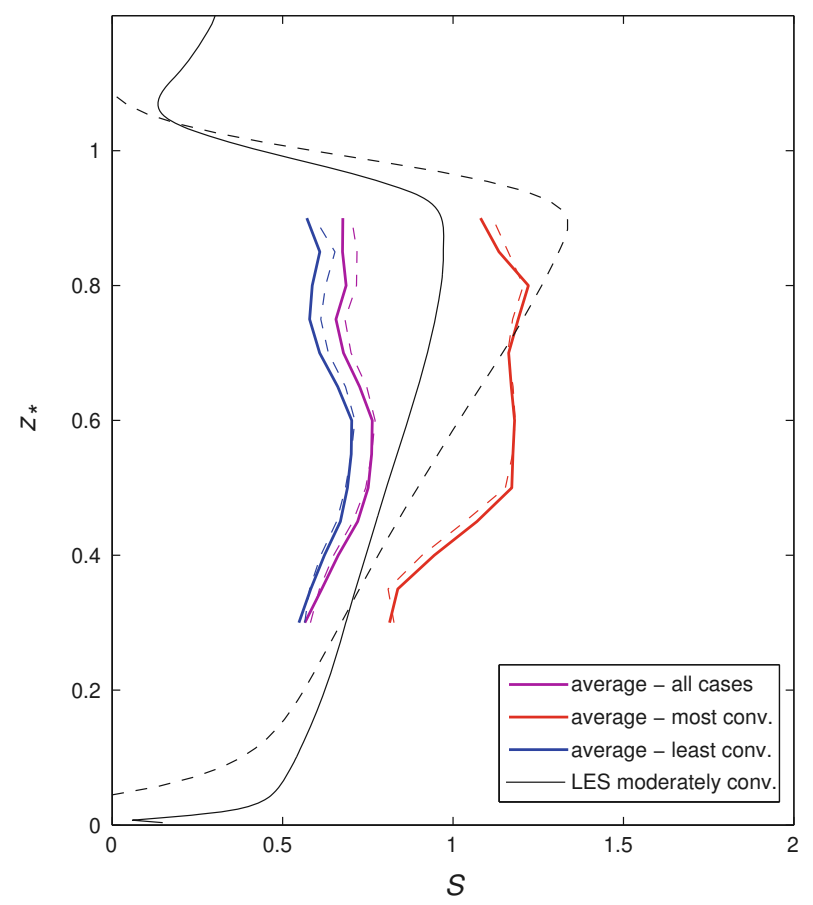

Fig. 9 Effect of spatial averaging on the $w$ skewness from LIFT observations and the effect of reduced resolution on LES—both are indicated by the dashed lines

$$
\tilde{w}(t) \equiv \tilde{z}(t)+a \frac{\tilde{z}^{2}(t)-\left\langle\tilde{z}^{2}(t)\right\rangle}{\left\langle\tilde{z}^{2}\right\rangle^{1 / 2}},
$$

where $a$ is a parameter that determines the departure of $\tilde{w}(t)$ from a Gaussian distribution. The skewness and kurtosis of this modified Gaussian process are

$$
\begin{aligned}
\tilde{S} & =\frac{2 a\left(3+4 a^{2}\right)}{\left(1+2 a^{2}\right)^{3 / 2}}, \\
\tilde{K} & =\frac{3\left(1+20 a^{2}+20 a^{4}\right)}{\left(1+2 a^{2}\right)^{2}} .
\end{aligned}
$$

They found that the parametric plot of $\tilde{S}(a)$ versus $\tilde{K}(a)$ from Eq. (6) was a reasonable fit to the distributions of aircraft $w$ and temperature measurements in the CBL. We see in Fig. 11 that this is a reasonable fit to the observations reported here.

Another relationship between $S$ and $K$ has been proposed by Gryanik and Hartmann (2002) and Gryanik et al. (2005), viz.

$$
K=a\left(b S^{2}+1\right),
$$

where $a$ and $b$ are constants. This result is based on a two-scale mass-flux model for the CBL. The basic assumption for this is that convection is characterized by buoyant updrafts (thermals) driven by surface heating and compensating downdrafts. From theoretical considerations, they initially suggested the values $a=3$ and $b=1 / 3$, but found that a better fit was obtained to their aircraft observations with the values $a=2.85$ and $b=0.505$. They 


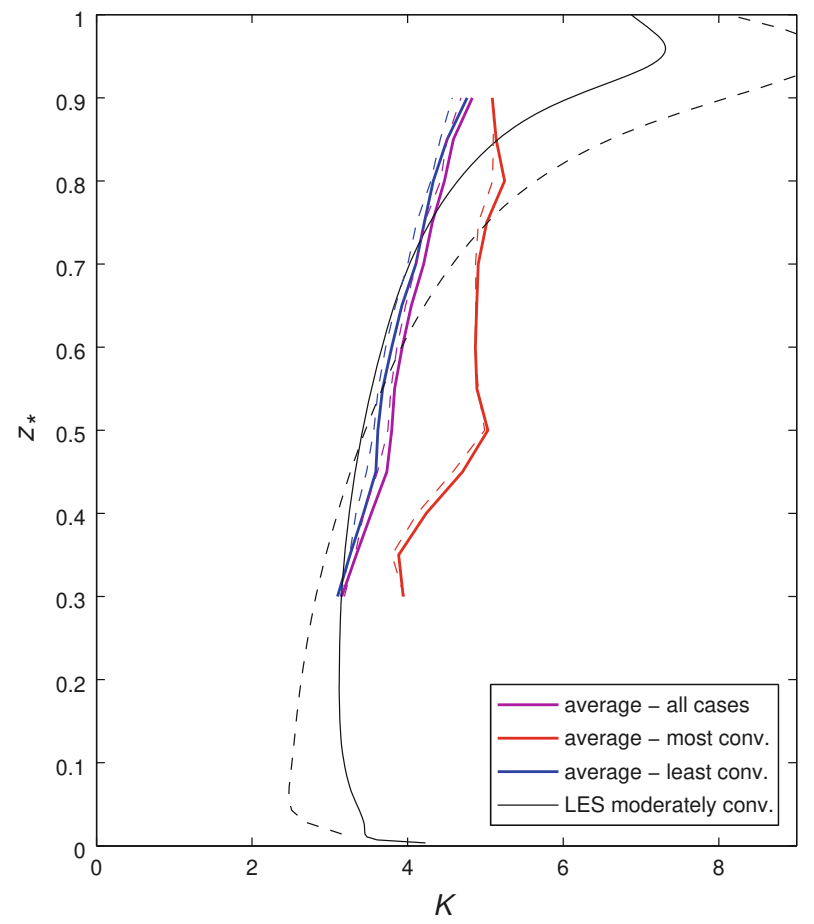

Fig. 10 Effect of spatial averaging on the $w$ kurtosis from LIFT observations and the effect of reduced resolution on LES - both indicated by the dashed lines

also found good agreement between their observations and LES results. We see in Fig. 11 that using the observationally adjusted coefficients in (7) gives a very similar curve to that of Lenschow et al. (1994), and that they both agree reasonably well with the observations. The LES results show less kurtosis than the observations for $S<0.8$, and somewhat more for $S>1.2$ - the LES results show a greater rate of increase of $K$ with increasing $S$ than the observations. This means that large intermittency in the observations, which is characteristic of the upper part of the CBL in the most convective cases, is accompanied by larger $S$ than in the LES results that flatten out for large $K$. The least convective (most shear) LES case is somewhat closer to the models and observations than is the more convective (least shear) case.

\subsection{Thermal Model}

The assumption of Gryanik et al. (2005) is similar to that of Weil et al. (1997), who assumed a probability distribution for $w$ that is the superposition of two Gaussian distributions-one that characterizes the updrafts and the other the downdrafts. They used this principle to construct a model for vertical dispersion in the CBL that showed reasonable agreement with convection tank data and observational results. We explore this approach further here by constructing a model of $S$ and $K$ based on a model that apportions the $w$ field obtained from aircraft measurements in a strongly convective marine CBL into thermals and their environment, and quantifies both the mean and variance of thermals (denoted by the subscript ()$_{\mathrm{T}}$ ) and their environment (denoted by the subscript ( )E) (Lenschow and Stephens 1982). The thermals, which were identified by a threshold value of humidity, which exceeded the mean 


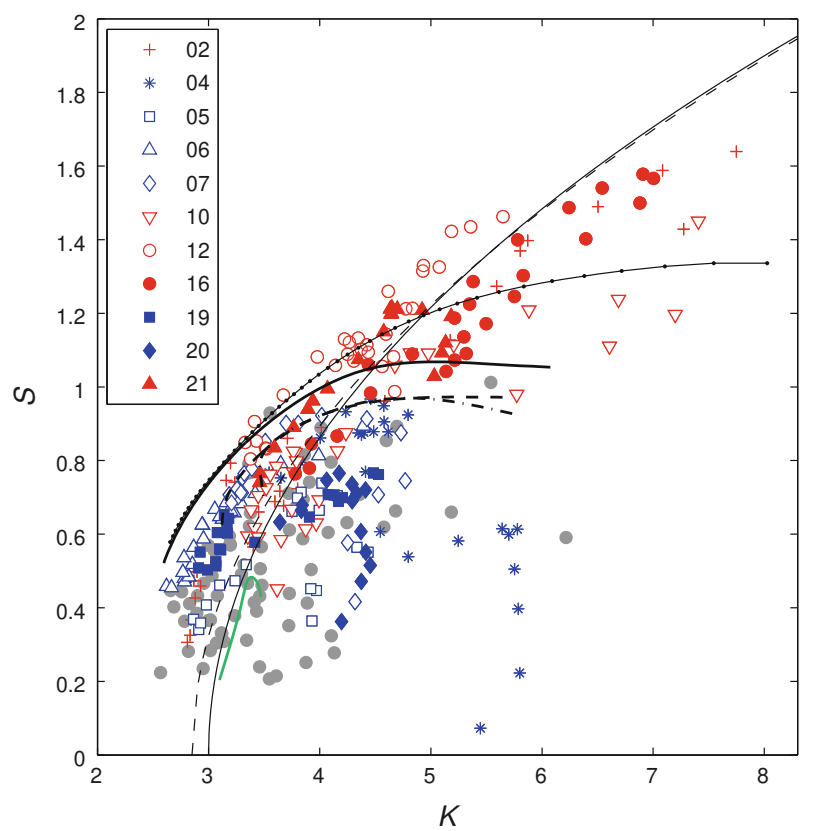

Fig. 11 Parametric curve of $S$ versus $K$ given by Eq. (6) (thin solid line), Gryanik and Hartmann (2002) model of $S$ versus $K$ given by Eq. (7 (thin dashed line), and thermal model of $S$ versus $K$ (green line). The coloured symbols are LIFT measurements. The filled grey circles are airplane measurements from AMMA. The thick black curves are LES simulations from Sullivan and Patton (2011), with the solid curve being the most convective case the dashed line the moderately convective case, and the dot-dash line the least convective case. The solid line punctuated with circles is the moderately convective case with reduced resolution

by half a standard deviation, were found to encompass about $28 \%$ and the environment about $72 \%$ of the total field. The means and standard deviations are

$$
\begin{aligned}
\bar{w}_{\mathrm{T}} / w_{*} & =1.02 z_{*}^{1 / 3}\left(1-1.1 z_{*}\right), \\
\sigma_{\mathrm{T}}^{2} / w_{*}^{2} & =2.2 z_{*}^{2 / 3}\left(1-2.18 z_{*}^{1 / 2}+1.82 z_{*}-0.55 z_{*}^{2}\right), \\
\bar{w}_{\mathrm{E}} / w_{*} & =-0.39 z_{*}^{1 / 3}\left(1-1.1 z_{*}\right), \\
\sigma_{\mathrm{E}}^{2} / w_{*}^{2} & =2.2 z_{*}^{2 / 3}\left(1-1.64 z_{*}^{1 / 4}+0.63 z_{*}^{1 / 2}+0.15 z_{*}-0.08 z_{*}^{2}\right) .
\end{aligned}
$$

We assume that the distributions of $w$ in both the thermals and their environment are Gaussian. The intention is to see how well a Gaussian assumption can represent the distributions in both the thermals and their environment. We then add the two distributions for the variances, each offset by their respective normalized mean velocities, $\bar{w}_{\mathrm{T}} / w_{*}$ and $\bar{w}_{\mathrm{E}} / w_{*}$, and solve for $S$ and $K$. We refer to this as the thermal model. The results are shown in Figs. 5, 7, and 11. We see that this model can reasonably describe $S$ only for $z_{*}<0.1$ and $K$ observations for $z_{*}<0.5$, but utterly fails above these levels, as $S$ reverses direction and decreases, while $K$ approaches a nearly constant value. This is because the thermals lose definition in the upper part of the CBL, with the means decreasing more rapidly than the variances, so that $S$ and $K$ both decrease and approach their Gaussian values. This indicates that the assumption of Gaussianity for both thermals and environment is reasonable only in the lower part of the CBL and is not a valid assumption above this. In the upper part of the CBL, the contribution of entrained air from the overlying free atmosphere is likely an important factor in causing this failure. 
An interesting aspect of this is that if we use the surface-layer observations reported by Wyngaard and Cote (1971) for $\left\langle w^{3}\right\rangle$ and Wyngaard et al. (1971) for $\left\langle w^{2}\right\rangle$, then we obtain $S \approx 0.25$. The thermal model gives $S \approx 0.06$ as $z_{*} \rightarrow 0$ and increases up to about 0.3 at $z_{*}=0.01$.

\section{Conclusions}

We have shown that the HRDL offers a new tool to obtain long-term averages of the vertical profiles of second- to fourth-order moments of $w$ throughout the mid-region of the CBL. The results show more vertical resolution of the behaviour of these moments than previously documented. The LIFT observations are also compared with observations from previous studies as well as LES results. The previous observations are in general agreement with our observations. The LES results are mostly in agreement with the observations, but both $S$ and $K$ show less dependency on stability than the observations, and flatter curves for $S$ versus $K$-smaller $K$ for smaller $S$ and larger $K$ for larger $S$. In fact, for small $S$ in the most convective case, the LES gives $K<3$, i.e. the distribution has fewer points at the extremes of the distribution than a Gaussian distribution. We speculate that the smaller LES estimates of $K$, especially in the surface layer, are a consequence of the subgrid-scale model that approximates the subgrid-scale fluxes and variances with a Gaussian approximation (Hunt et al. 1988).

More detail close to the surface might be achieved if the HRDL 'dead zone' could be reduced, and extension to higher levels, including the entrainment zone and the free troposphere, might be achieved if backscatter sensitivity could be increased. This would add further value to the HRDL as a tool for gathering long-term turbulence statistics in the atmospheric boundary layer.

Acknowledgements The LIFT experiment was funded by the NCAR Earth Observatory Laboratory Director's Office and the Department of Energy/OAGR. The authors express their thanks to the MMM Division and ATD of NCAR for their help during this study. The National Center for Atmospheric Research is sponsored by the National Science Foundation.

\section{References}

Angevine WM, Grimsdell AW, Hartten LM, Delany AC (1998) The flatland boundary layer experiments. Bull Amer Meteorol Soc 79:419-431

Ansmann A, Fruntke J, Engelmann R (2010) Updraft and downdraft characterization with Doppler lidar: cloud-free versus cumuli-topped mixed layer. Atmos Chem Phys 10:7845-7858

Canut G, Lothon M, Said F (2010) Observation of entrainment at the interface between monsoon flow and saharan air layer. Q J R Meteorol Soc 136(s1):34-46

Cohn SA, Mayor SD, Grund TM, Weckwerth TM, Senff C (1998) The lidars in flat terrain experiment (LIFT). Bull Am Meteorol Soc 79:1329-1343

Grund CJ, Banta RM, George J, Howell JN, Post MJ, Richter RA (1998) High-resolution Doppler lidar for boundary layer and cloud research. J Atmos Ocean Technol 18:376-393

Gryanik VM, Hartmann J (2002) A turbulence closure for the convective boundary layer based on a two-scale mass-flux approach. J Atmos Sci 59:2729-2744

Gryanik VM, Hartmann J, Raasch S, Schröter M (2005) A refinement of the millionshchikov quasi-normality hypothesis for convective boundary layer turbulence. J Atmos Sci 62:2632-2638

Hicks B, Wesely M (1981) Heat and momentum transfer characteristics of adjacent fields of soybeans and maize. Boundary-Layer Meteorol 20:175-185

Hunt JCR, Kaimal JC, Gaynor JE (1988) Eddy structure in the convective boundary layer-new measurements and new concepts. Q J R Meteorol Soc 482:827-858 
Kaimal JC, Wyngaard JC, Haugen DA, Coté OR, Izumi Y (1976) Turbulence structure in the convective boundary layer. J Atmos Sci 33:2152-2169

Lenschow DH, Stephens PL (1982) The role of thermals in the convective boundary layer. Atmos Environ 16:761-764

Lenschow DH, Wyngaard JC, Pennell WT (1980) Mean-field and second-moment budgets in a baroclinic, convective boundary layer. J Atmos Sci 37:1313-1326

Lenschow DH, Mann J, Kristensen L (1994) How long is long enough when measuring fluxes and other turbulence statistics?. J Atmos Ocean Technol 11:661-673

Lenschow DH, Wulfmeyer V, Senff C (2000) Measuring second- through fourth-order moments in noisy data. J Atmos Ocean Technol 17:1330-1347

Lothon M, Lenschow DH, Mayor SD (2006) Coherence and scale of vertical velocity in the convective boundary layer from a Doppler lidar. Boundary-Layer Meteorol 121:521-536

Lothon M, Lenschow DH, Mayor SD (2009) Doppler lidar measurements of vertical velocity spectra in the convective boundary-layer. Boundary-Layer Meteorol 132:205-226

Militzer JM, Michaelis MC, Semmer SR, Norris KS, Horst TW, Oncley SP, Delany AC, Brock FV (1995) Development of the prototype pamiii/flux-pam surface meteorological station. In: Proceedings of the 9th symposium on meteorological observations and instrumentation. American Meteorological Society, Boston, pp 490-494

Moeng CH (1984) A large-eddy simulation model for the study of planetary boundary-layer turbulence. J Atmos Sci 41:2052-2062

Moeng CH, Rotunno R (1990) Vertical velocity skewness in the buoyancy-driven boundary layer. J Atmos Sci 47:1149-1162

Moeng CH, Wyngaard JC (1988) Spectral analysis of large-eddy simulations of the convective boundary layer. J Atmos Sci 45:3573-3587

O'Connor EJ, Illingworth AJ, Brooks IM, Westbrook CD, Hogan RJ, Davies F, Brooks BJ (2010) A method for estimating the turbulent kinetic energy dissipation rate from a vertically pointing Doppler lidar, and independent evaluation from balloon-borne in situ measurements. J Atmos Ocean Technol 27:1652-1664

Redelsperger JL, Thorncroft C, Diedhiou A, Lebel T, Parker DJ, Polcher J (2006) African Monsoon Multidisciplinary Analysis (AMMA): an international research project and field campaign. Bull Am Meteorol Soc 87:1739-1746

Sullivan PP, Patton EG (2011) The effect of mesh resolution on convective boundary-layer statistics and structures generated by large-eddy simulation. J Atmos Sci (in press)

Sullivan PP, McWilliams JC, Moeng CH (1994) A subgrid-scale model for large-eddy simulation of planetary boundary layer flows. Boundary-Layer Meteorol 71:247-276

Sullivan PP, McWilliams JC, Moeng CH (1996) A grid nesting method for large-eddy simulation of planetary boundary-layer flows. Boundary-Layer Meteorol 80:167-202

Tennekes H (1973) A model for the dynamics of the inversion above a convective boundary layer. J Atmos Sci 30:558-567

Weckwerth T (1999) An observational study of the evolution of horizontal convective rolls. Mon Weather Rev 127:2160-2179

Weil JC, Corio LA, Brower RP (1997) A PDF dispersion model for buoyant plumes in the convective boundary layer. J Appl Meteorol 36:982-1003

Wyngaard J (1988) Structure of the PBL. In: Venkatram A, Wyngaard J (eds) Lectures on air pollution modeling. American Meteorological Society, Boston

Wyngaard JC, Cote O (1971) The budgets of turbulent kinetic energy and temperature variance in the atmospheric surface layer. J Atmos Sci 28:190-201

Wyngaard JC, Cote O, Izumi Y (1971) Local free convection, similarity, and the budgets of shear stress and heat flux. J Atmos Sci 41:1171-1182 